\title{
Battery lifetime and PSNR quality-scalable video transmission for mobile multimedia systems
}

\author{
Seongsoo Lee ${ }^{\text {a) }}$ and Yongju Jang \\ School of Electronic Engineering, Soongsil University, \\ 369 Sangdo-ro, Dongjak-gu, Seoul 156-743, Korea \\ a) sslee@ssu.ac.kr
}

\begin{abstract}
A battery lifetime and PSNR quality-scalable video transmission is proposed. Video bitstream is intentionally encoded at lowered bitrate, and it is intermittently transmitted to exploit battery recovery effect. Battery lifetime is extended significantly with PSNR quality degradation given by user. Both battery lifetime and PSNR quality are precisely estimated and easily controlled by adjusting encoding bitrate and battery duty ratio. Battery lifetime extension is fully scalable with PSNR quality degradation, and vice versa. Measured battery lifetime was extended up to 31.4 times at the cost of 1.20 3.89 dB PSNR degradation in H.264 video coding and ZigBee transceiver. Absolute average errors of estimated PSNR degradation and battery lifetime were less than $7.31 \%$.
\end{abstract}

Keywords: video coding, video transmission, battery recovery effect, battery lifetime extension, scalability, PSNR quality, battery lifetime

Classification: Electron devices, circuits, and systems

\section{References}

[1] Z. He, W. Cheng and X. Chen: IEEE Trans. Circuits Syst. Video Technol. 18 (2008) 596. DOI:10.1109/TCSVT.2008.918802

[2] S. Sharangi, R. Krishnamurti and M. Hefeeda: IEEE Trans. Multimed. 13 (2011) 102. DOI:10.1109/TMM.2010.2076799

[3] V. Rao, G. Singhal, A. Kumar and N. Navet: Proc. IEEE Int. Conf. VLSI Des. (2005) 105. DOI:10.1109/ICVD.2005.61

[4] Y. Lee, J. Kim and C. Kyung: IEEE Trans. Very Large Scale Integr. (VLSI) Syst. 20 (2012) 310. DOI:10.1109/TVLSI.2010.2102055

[5] C. Chau, F. Qin, S. Sayed, M. Wahab and Y. Yang: IEEE J. Sel. Areas Commun. 28 (2010) 1222. DOI:10.1109/JSAC.2010.100926

[6] Y. Jang, H. Yang and S. Lee: Proc. IEEE Int. Conf. Consum. Electr. (2012) 414. DOI:10.1109/ICCE.2012.6161954

[7] ISO/IEC: ITU-T Rec. H.264/ISO/IEC 14496-10 AVC (2003).

[8] F. De Vito and J. De Martin: Proc. IEEE Int. Symp. Signal Process. Inf. Technol. (2005) 612. DOI:10.1109/ISSPIT.2005.1577167

[9] L. Teixeira: in Advances on Video Coding, ed. J. Lorente (Intech, 2011) 133. 


\section{Introduction}

Battery lifetime is one of the most critical issues in mobile multimedia systems. In general, there is a trade-off between power consumption and peak-signal-to-noiseratio (PSNR) quality. Various methods $[1,2]$ have been proposed to optimize them. However, they cannot significantly extend nor easily estimate the battery lifetime, since they do not consider an important characteristics of the battery.

As illustrated in Fig. 1(a), battery voltage increases when the battery is intermittently discharged by shutting down the target system during some intervals. Thus, battery lifetime of intermittent discharge is longer than that of continuous discharge even if their power consumptions are same. This is called as battery recovery effect [3], and it significantly extends the battery lifetime.

[4] and [5] have been proposed to consider battery recovery effect in the modeling of battery lifetime in mobile multimedia systems. However, they exploit battery recovery effect indirectly, so more aggressive methods are necessary.

[6] has been proposed to further exploit battery recovery effect. It controls the video encoding bitrate to give some duty cycles to the wireless transmitter. It extends the battery lifetime while sacrificing PSNR quality. However, it is difficult to achieve quality-power scalability, since it has no estimation models for battery lifetime and PSNR quality. Furthermore, it shutdowns only wireless transmitter, so it can be applied to some limited cases only when encoder power is negligible in comparison with transmitter power.

In this Letter, a battery Lifetime and PSNR Quality-Scalable Video Transmission (LQSVT) is proposed based on [6]. It significantly extends battery lifetime using battery recovery effect. Both battery lifetime and PSNR quality are easily controlled with full scalability using simple but precise estimation models and control methods. Furthermore, power consumption of the video encoder is fully considered, so it can be practically applied to mobile multimedia systems.

\section{The proposed method}

\subsection{Intermittent video transmission}

In the conventional video transmission, encoding and transmission bitrates are $\mathrm{BR}_{\mathrm{REF}}$ as illustrated in Fig. 1(b). In the proposed LQSVT, intermittent transmission is performed to exploit battery recovery effect, as illustrated in Fig. 1(c). Encoding bitrate is decreased to $\mathrm{BR}_{\mathrm{TAR}}$, while transmission bitrate remains unchanged. Battery lifetime is significantly extended by two effects. First, system power is decreased since its active time is decreased. Then, battery recovery effect further extends the battery lifetime. However, PSNR quality is degraded due to the decreased encoding bitrate.

In the LQSVT, two important parameters are introduced. Battery lifetime extension ratio (BLER) is defined as $\mathrm{BLER}_{\mathrm{TAR}}=\mathrm{LT}_{\mathrm{TAR}} / \mathrm{LT}_{\mathrm{REF}}$, and it is a performance measure of LQSVT. Battery duty ratio $(\mathrm{DR})$ is defined as $\mathrm{DR}_{\mathrm{TAR}}=$ $\mathrm{T}_{\mathrm{A}} / \mathrm{T}_{\mathrm{P}}$ and it controls intermittent discharge.

Duty ratio is determined as illustrated in Fig. 1(d). In the LQSVT, both video encoder and wireless transmitter simultaneously become active and shutdown with same duty ratio. Transmitter power highly depends on the encoding bitrate, since it 


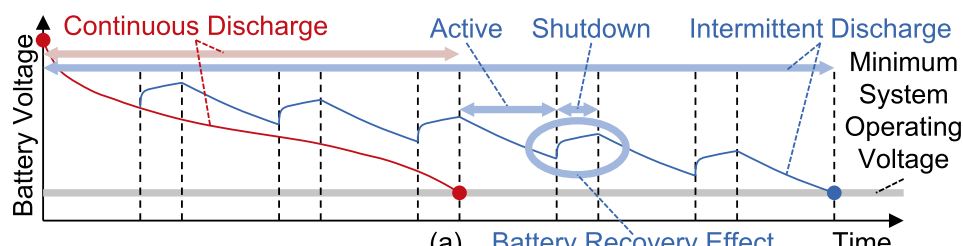

(a) Battery Recovery Effect Time
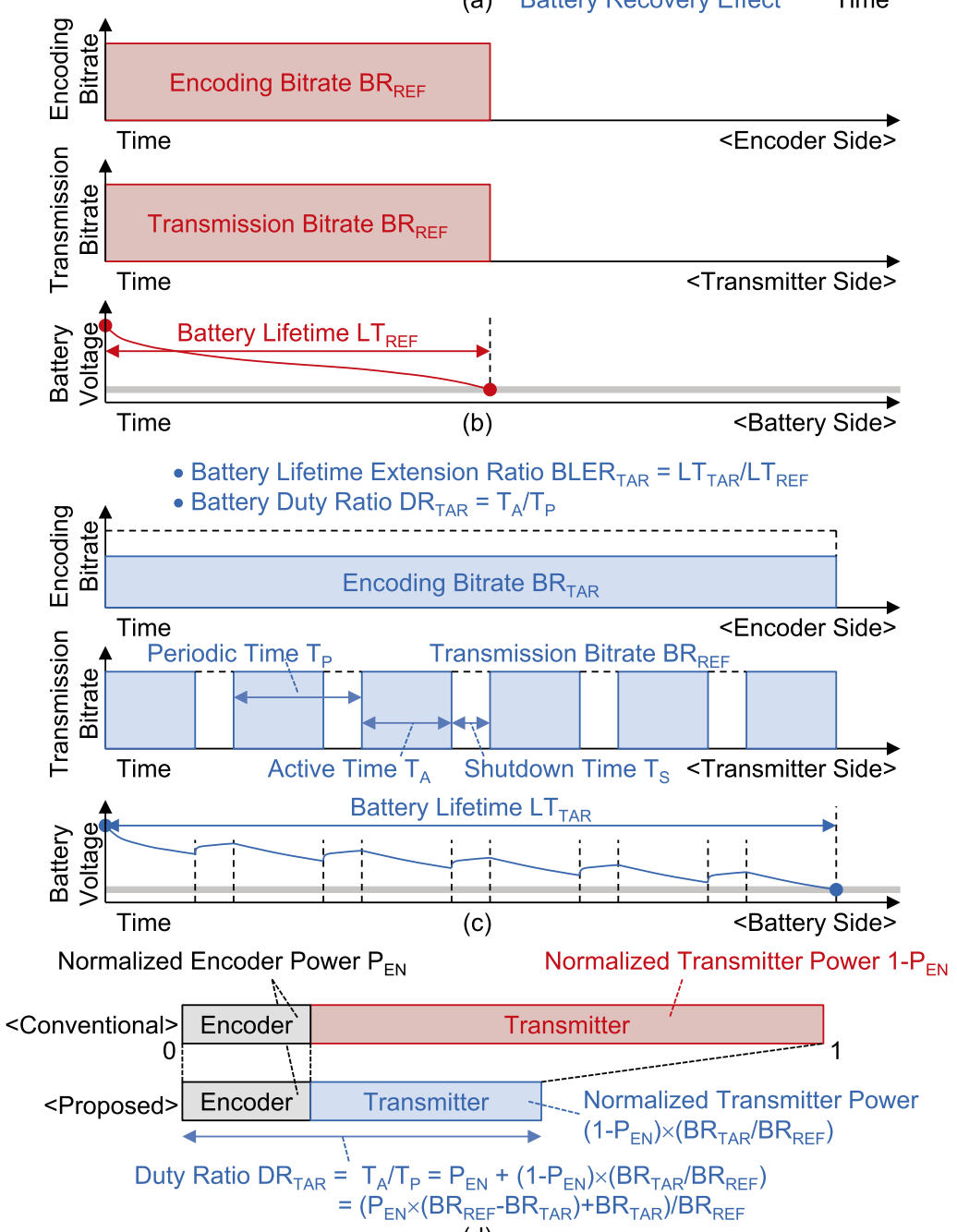

(d)

Fig. 1. The proposed LQSVT method. (a) Intermittent discharge and battery recovery effect. (b) Conventional video transmission. (c) Proposed video transmission. (d) Determination of battery duty ratio.

is roughly proportional to the active transmission time. However, encoder power is almost unchanged. Considering this, duty ratio is determined as $\mathrm{DR}_{\mathrm{TAR}}=\mathrm{P}_{\mathrm{EN}}+$ $\left(1-\mathrm{P}_{\mathrm{EN}}\right) \times\left(\mathrm{BR}_{\mathrm{TAR}} / \mathrm{BR}_{\mathrm{REF}}\right)=\left(\mathrm{P}_{\mathrm{EN}} \times\left(\mathrm{BR}_{\mathrm{REF}}-\mathrm{BR}_{\mathrm{TAR}}\right)+\mathrm{BR}_{\mathrm{TAR}}\right) / \mathrm{BR}_{\mathrm{REF}}$, where the normalized encoder power is $\mathrm{P}_{\mathrm{EN}}=$ encoder power/(encoder power + transmitter power) without shutdown. Note that $\mathrm{DR}_{\mathrm{TAR}}=\mathrm{BR}_{\mathrm{TAR}} / \mathrm{BR}_{\mathrm{REF}}$ when $\mathrm{P}_{\mathrm{EN}}=0$, since transmitter power is proportional to encoding bitrate $\mathrm{BR}_{\mathrm{TAR}}$ when encoder power is negligible. Also note that $\mathrm{DR}_{\mathrm{TAR}}=1$ when $\mathrm{P}_{\mathrm{EN}}=1$, since only non-scalable encoder power remains when transmitter power is negligible. 


\subsection{Control of battery lifetime and PSNR quality}

In most video coding algorithms, target bitrate controls quantization parameter (QP), and QP deeply affects PSNR quality. So there are close relations between PSNR $\leftrightarrow$ QP $\leftrightarrow$ bitrate.

As illustrated in Section 2.1, duty ratio is a function of bitrate and BLER is a function of lifetime. Furthermore, duty ratio deeply affects BLER. So there are close relations between bitrate $\leftrightarrow$ duty ratio $\leftrightarrow$ BLER $\leftrightarrow$ lifetime.

From above, there are close relations between PSNR $\leftrightarrow$ QP $\leftrightarrow$ bitrate $\leftrightarrow$ duty ratio $\leftrightarrow$ BLER $\leftrightarrow$ lifetime. It means, setting the amount of PSNR degradation can control the amount of battery lifetime extension with scalability, and vice versa, if proper estimation models and control methods are provided.

As illustrated in Fig. 2, LQSVT proposes two control modes, i.e. PSNR quality-scalable and battery lifetime-scalable modes. The former can be used when PSNR quality is important, e.g. video surveillance. The latter can be used when battery lifetime is important, e.g. watching live sport games. Note that these two modes are almost same, so a user can easily switch them in the same device.

In PSNR quality-scalable mode, a user scales PSNR quality by giving acceptable PSNR degradation limit. LQSVT keeps the PSNR degradation close to this target value while maximizing the battery lifetime extension. Also, expected battery lifetime can be optionally reported to the user. Battery lifetime-scalable mode is almost same, except PSNR degradation and battery lifetime are exchanged.
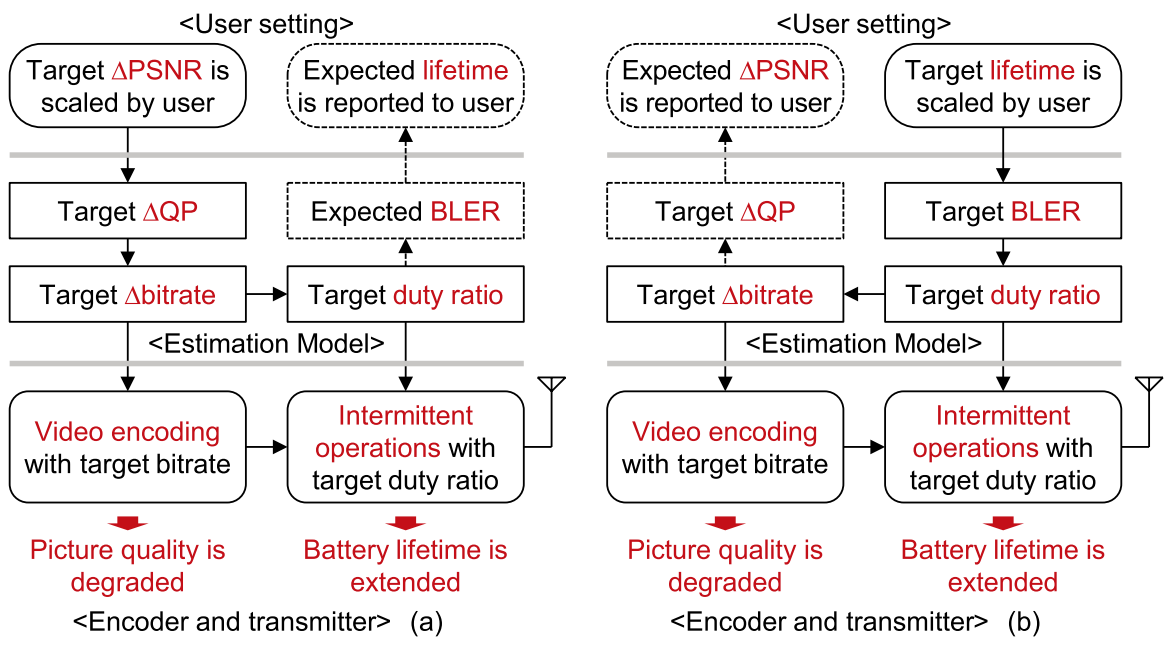

Fig. 2. Scalability control modes. (a) PSNR quality-scalable. (b) Battery lifetime-scalable.

\section{The estimation models}

\subsection{Video encoder estimation models (PSNR $\leftrightarrow$ QP $\leftrightarrow$ bitrate)}

To develop these models, H.264 video coding [7] was performed on two typical QCIF test sequences. Reference simulation results were shown in Figs. 3(a)-(c). Target PSNR degradation, target QP increment, and target bitrate decrement are defined as $\triangle \mathrm{PSNR}_{\mathrm{TAR}}=\mathrm{PSNR}_{\mathrm{REF}}-\mathrm{PSNR}_{\mathrm{TAR}}, \Delta \mathrm{QP}_{\mathrm{TAR}}=\mathrm{QP}_{\mathrm{TAR}}-\mathrm{QP}_{\mathrm{REF}}$, and $\Delta \mathrm{BR}_{\mathrm{TAR}}=\mathrm{BR}_{\mathrm{REF}}-\mathrm{BR}_{\mathrm{TAR}}$, respectively. 

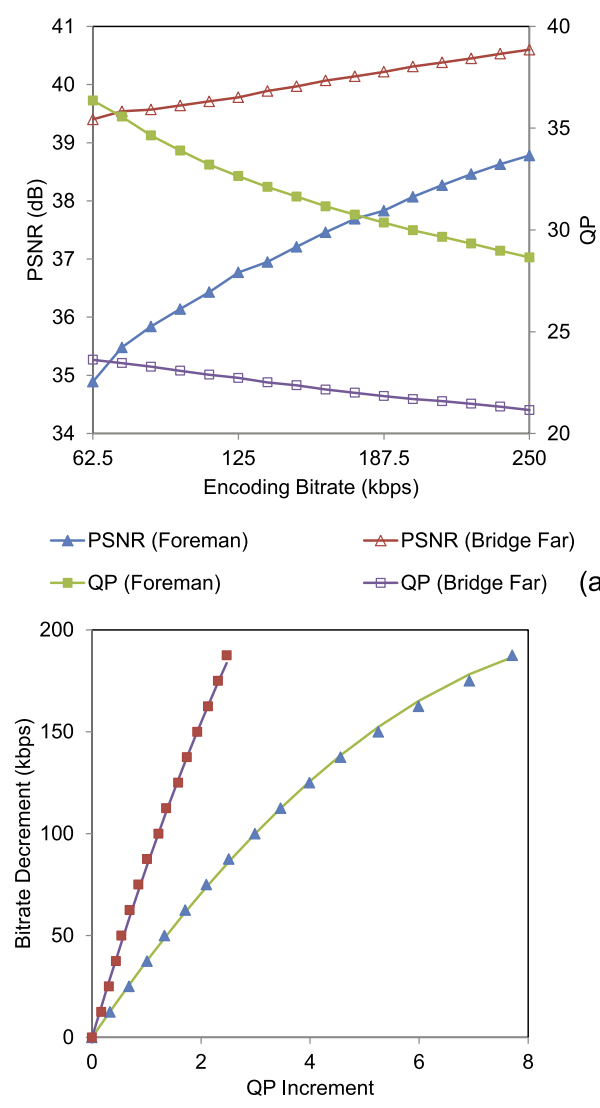

Measured Bitrate Decrement (Foreman) Measured Bitrate Decrement (Bridge Far) Estimated Bitrate Decrement (Foreman) stimated Bitrate Decrement (Brid

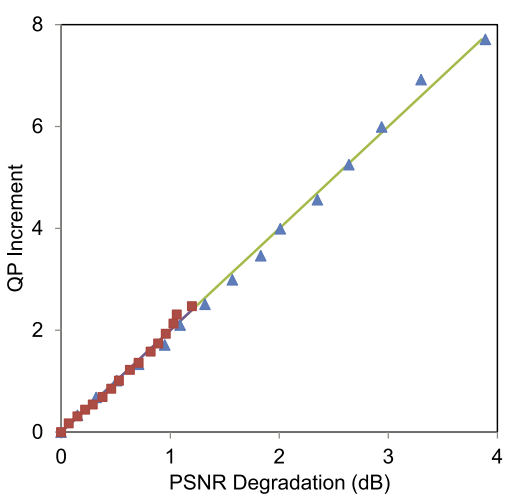

A Measured QP Increment (Foreman) - Measured QP Increment (Bridge Far) Estimated QP Increment (Foreman)

(a) (b)

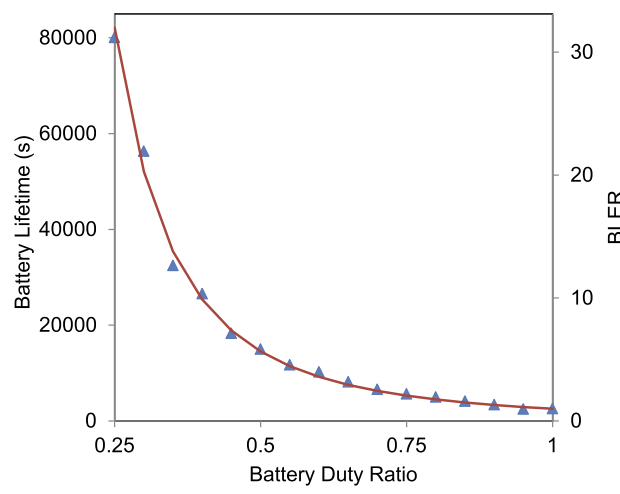

(c) $\triangle$ Measured Battery Lifetime \& BLER

-Estimated Battery Lifetime \& BLER

Fig. 3. Reference simulation and measurement results. (a) PSNR, $\mathrm{QP}$, and bitrate. (b) $\triangle \mathrm{QP}_{\mathrm{TAR}}$ as a function of $\triangle \mathrm{PSNR}_{\mathrm{TAR}}$. (c) $\triangle \mathrm{BR}_{\mathrm{TAR}}$ as a function of $\Delta \mathrm{QP}_{\mathrm{TAR}} \cdot(\mathrm{d}) \mathrm{LT}_{\mathrm{TAR}}$ and BLER $\mathrm{TAR}$ as functions of $\mathrm{DR}_{\mathrm{TAR}}$.

In [8], $\triangle \mathrm{QP}_{\mathrm{TAR}}$ can be modeled as a linear function of $\triangle \mathrm{PSNR}_{\mathrm{TAR}}$. $\Delta \mathrm{QP}_{\mathrm{TAR}}=0$ when $\Delta \mathrm{PSNR}_{\mathrm{TAR}}=0$, so $\Delta \mathrm{QP}_{\mathrm{TAR}}=\mathrm{A} \times \Delta \mathrm{PSNR}_{\mathrm{TAR}}$ where $\mathrm{A}$ is a model parameter. Similarly, in [9], $\Delta \mathrm{BR}_{\mathrm{TAR}}$ can be modeled as a second-order polynomial function of $\Delta \mathrm{QP}_{\mathrm{TAR}} \cdot \Delta \mathrm{BR}_{\mathrm{TAR}}=0$ when $\Delta \mathrm{QP}_{\mathrm{TAR}}=0$, so $\Delta \mathrm{BR}_{\mathrm{TAR}}=$ $\mathrm{B} \times \Delta \mathrm{QP}_{\mathrm{TAR}}+\mathrm{C} \times \Delta \mathrm{QP}_{\mathrm{TAR}}{ }^{2}$, where model parameters $\mathrm{B}$ and $\mathrm{C}$ are functions of $\mathrm{QP}_{\mathrm{REF}}$ as in [10].

From above, the estimation models between PSNR $\leftrightarrow$ QP $\leftrightarrow$ bitrate are developed as Eqs. (1) and (2), where model parameters were obtained by curve fitting. Note that $\left(\mathrm{QP}_{\mathrm{REF}}\right)^{3.82}$ is not a variable but a constant obtained from reference simulation. Figs. 3(b) and (c) show that the proposed estimation models are precise enough for practical use.

$$
\begin{aligned}
& \Delta Q P_{T A R}=2 \times \Delta P S N R_{T A R} \\
& \Delta B R_{T A R}=\left(\frac{8500000}{Q P_{R E F}^{3.82}}+16.2\right) \times \Delta Q P_{T A R}-\frac{720000}{Q P_{R E F}^{3.82}} \times \Delta Q P_{T A R}^{2}
\end{aligned}
$$




\subsection{Battery discharge estimation models (bitrate $\leftrightarrow$ duty ratio $\leftrightarrow$ BLER $\leftrightarrow$ lifetime)}

To develop these models, a battery-operated wireless transmission environment was established using a ZigBee transceiver and a $\mathrm{LiMnO}_{2}$ battery. Reference measurement results were shown in Fig. 3(d).

Fig. 3(d) indicates that BLER $\mathrm{TAR}_{\mathrm{TAR}}$ can be modeled as an exponential function of $\mathrm{DR}_{\mathrm{TAR}} \cdot \mathrm{BLER}_{\mathrm{TAR}}=1$ when $\mathrm{DR}_{\mathrm{TAR}}=1$, so $\mathrm{BLER}_{\mathrm{TAR}}=\left(\mathrm{DR}_{\mathrm{TAR}}\right)^{\mathrm{D}}$ where $\mathrm{D}$ is a model parameter. Also, in Section 2.1, $\mathrm{DR}_{\mathrm{TAR}}$ is modeled as a function of $\Delta \mathrm{BR}_{\mathrm{TAR}}$ and $\mathrm{LT}_{\mathrm{TAR}}$ is modeled as a function of BLER $\mathrm{TAR}_{\mathrm{T}}$.

From above, the estimation models between bitrate $\leftrightarrow$ duty ratio $\leftrightarrow$ BLER $\leftrightarrow$ lifetime are developed as Eqs. (3)-(5), whose model parameters were obtained by curve fitting. Note that $\mathrm{P}_{\mathrm{EN}}$ is not a variable but a constant from reference measurement. Fig. 3(d) shows that the propose estimation models are precise enough for practical use.

$$
\begin{aligned}
& D R_{T A R}=\frac{P_{E N} \times\left(B R_{R E F}-B R_{T A R}\right)+B R_{T A R}}{B R_{R E F}}=1-\frac{\left(1-P_{E N}\right)}{B R_{R E F}} \times \Delta B R_{T A R} \\
& B L E R_{T A R}=D R_{T A R}^{-2.5} \\
& L T_{T A R}=L T_{R E F} \times B L E R_{T A R}
\end{aligned}
$$

\section{Experimental results}

\subsection{Experimental environments}

Fig. 4(a) shows the experimental environments with a $2.4 \mathrm{GHz}$ ZigBee transceiver. Its active and shutdown currents were measured as $23 \mathrm{~mA}$ and $1 \mu \mathrm{A}$, respectively. DAQ monitored battery voltage to measure battery lifetime, as shown in Fig. 4(b). A $\mathrm{LiMnO}_{2}$ battery was used whose voltage and capacity are $3 \mathrm{~V}$ and $240 \mathrm{mAh}$, respectively. To mitigate battery cell deviation, initial voltages of all cells were measured and only cells with very similar initial voltages were used in the experiments. Furthermore, each experimental configuration was repeatedly measured by 80 times inside a metal-shielded chamber.

In the LQSVT, both video encoder and wireless transmitter should shutdown to fully exploit battery recovery effect. Many discrete ZigBee transceiver chips support fast shutdown and wakeup, but no such discrete video decoder chips are available to us. Therefore, in the experiments, encoder power was emulated by adding extra transmission of dummy data (thus extra power) in the transmitter. This is illustrated in Figs. 4(c) and (d).

Note that this emulation does not seriously affect the feasibility and usefulness of the LQSVT. Extra power in the transmission is almost equivalent to encoder power, and battery lifetime is affected by only total power. Also, there are no serious implementation issues, since many on-chip video encoder IPs already support shutdown and wakeup in mobile application processors.

H.264 standard video coding was performed on two typical QCIF $(176 \times 144$ pixels, 30 frames/s, 100 frames) test sequences, i.e. "Foreman" (close-up view with large motion) and "Bridge Far" (distance view with small motion). Reference encoding bitrate was $\mathrm{BR}_{\mathrm{REF}}=250 \mathrm{kbps}$, since it is the maximum transmission bitrate of ZigBee transceivers. From reference simulations and measurements, 


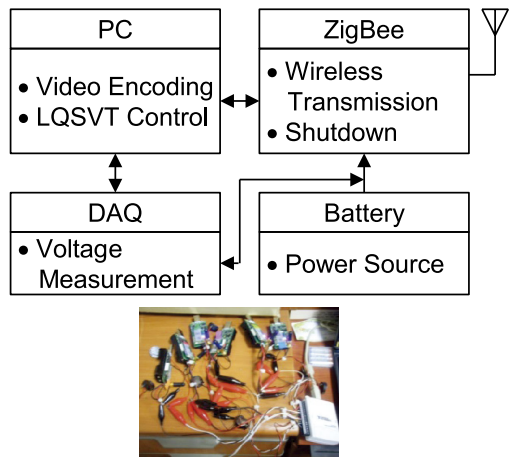

(a)

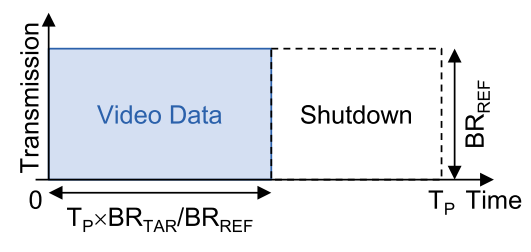

(c)
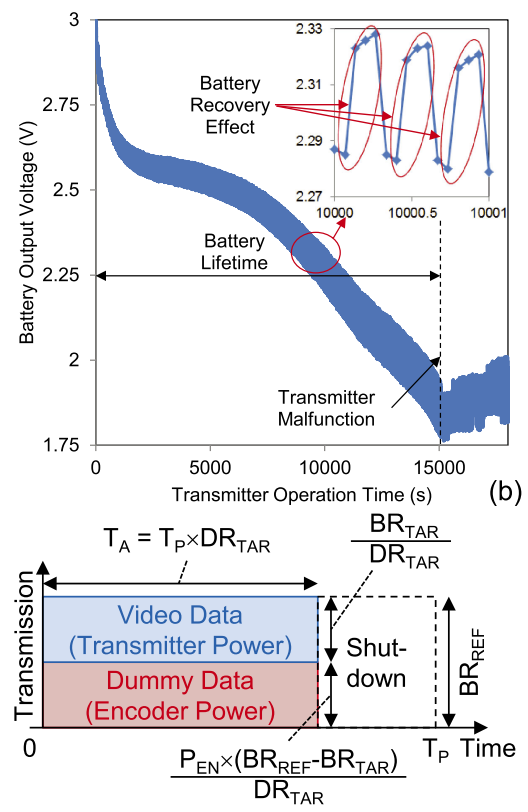

(d)

Fig. 4. Experimental environments and encoder power emulation.

(a) Measurement setup. (b) Battery output voltage monitoring.

(c) Transmitter operation with negligible encoder power.

(d) Transmitter operation considering encoder power.

reference PSNR quality, average QP, and reference battery lifetime were obtained as $\mathrm{PSNR}_{\mathrm{REF}}=38.78 \mathrm{~dB}$ (Foreman) \& $40.60 \mathrm{~dB}$ (Bridge Far), $\mathrm{QP}_{\mathrm{REF}}=28.65$ (Foreman) \& 21.15 (Bridge Far), and $\mathrm{LT}_{\mathrm{REF}}=2576 \mathrm{sec}$, respectively. Periodic time of intermittent discharge was $T_{P}=1 / 3 \mathrm{sec}$. Target bitrate was limited as $\mathrm{BR}_{\mathrm{TAR}} \geq 62.5 \mathrm{kbps}\left(1 / 4\right.$ of $\left.\mathrm{BR}_{\mathrm{REF}}\right)$, since too low bitrate leads to severe and impractical PSNR degradation.

\subsection{Measurement results in PSNR quality-scalable mode}

In the PSNR quality-scalable mode, LQSVT keeps the PSNR degradation close to $\triangle \mathrm{PSNR}_{\mathrm{TAR}}$ while maximizing BLER $\mathrm{TAR}_{\mathrm{T}}$ by controlling $\mathrm{DR}_{\mathrm{TAR}}$.

Fig. 5 shows the PSNR estimation error and battery lifetime with $\mathrm{P}_{\mathrm{EN}}=0$, i.e. encoder power is negligible. Absolute average estimation error of PSNR degradation was less than $3.22 \%$. Measured battery lifetime was extended by 31.2 times (Bridge Far) 31.4 times (Foreman). Fig. 6 shows the PSNR estimation error and battery lifetime with $\mathrm{P}_{\mathrm{EN}}=0.2$, i.e. encoder power is $20 \%$. Absolute average estimation error of PSNR degradation was less than $4.65 \%$. Measured battery lifetime was extended by 10.3 times (Bridge Far) 10.6 times (Foreman).

From the measurement results, PSNR degradation is fully scalable and precisely controlled to keep target constraint. Battery lifetime is also precisely estimated. When encoder power increases, BLER is less increased at the same PSNR degradation, since encoder power is not changed by LQSVT.

\subsection{Measurement results in battery lifetime-scalable mode}

In the battery lifetime-scalable mode, LQSVT keeps the lifetime extension close to BLER $_{\mathrm{TAR}}$ while minimizing $\triangle \mathrm{PSNR}_{\mathrm{TAR}}$ by controlling $\triangle \mathrm{QP}_{\mathrm{TAR}}$. Estimation models are inverse functions of Eqs. (1)-(5), which can be easily solved. 

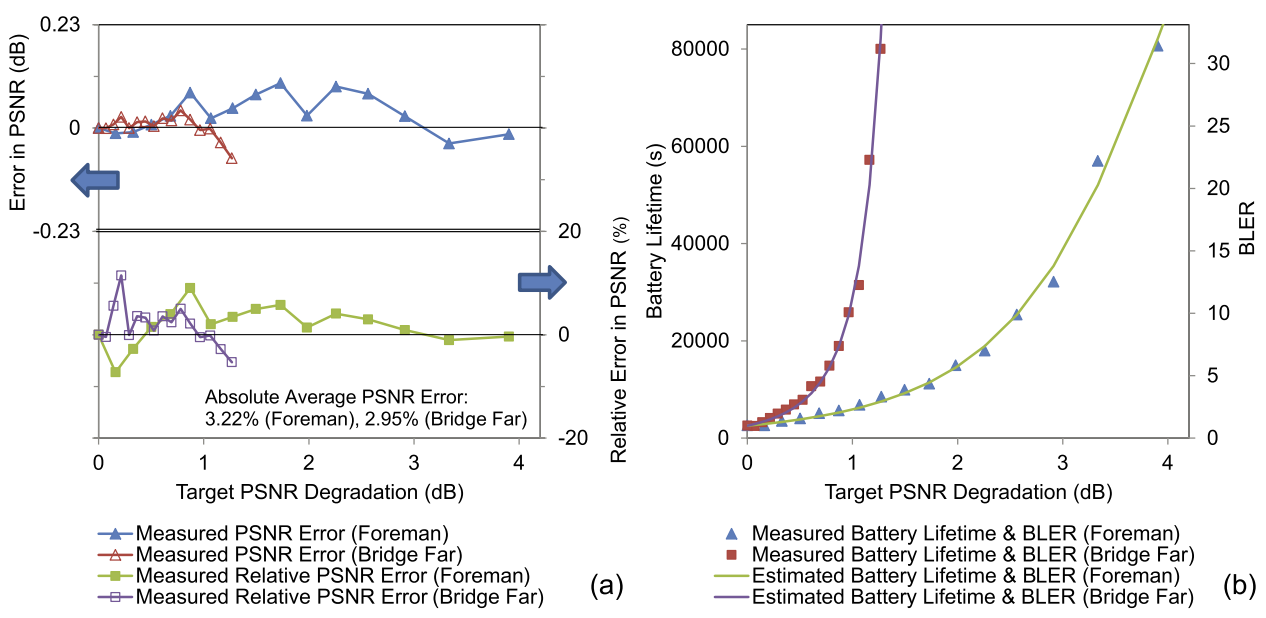

Fig. 5. Measurement results in PSNR quality-scalable mode with $\mathrm{P}_{\mathrm{EN}}=0$. (a) PSNR error. (b) Battery lifetime.

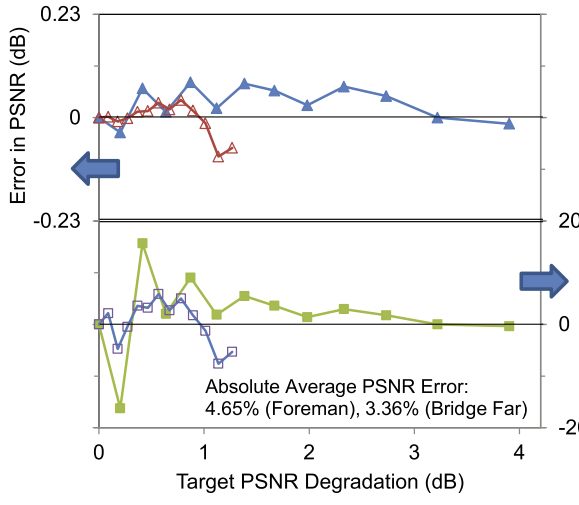

\-Measured PSNR Error (Foreman) $\triangle$ Measured PSNR Error (Bridge Far) - Measured Relative PSNR Error (Foreman)

Fig. 6. Measurement results in PSNR quality-scalable mode with $\mathrm{P}_{\mathrm{EN}}=0.2$ (a) PSNR error. (b) Battery lifetime.
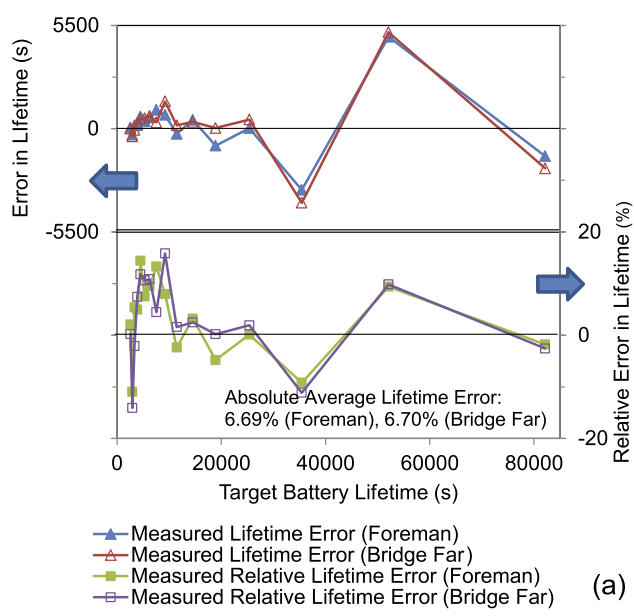

(a)

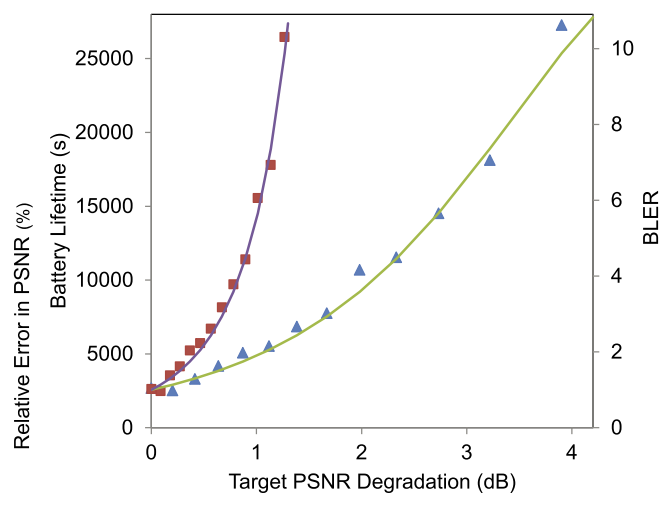

A Measured Battery Lifetime \& BLER (Foreman) - Measured Battery Lifetime \& BLER (Bridge Far)
-Estimated Battery Lifetime \& BLER (Foreman)
Estimated Battery Lifetime \& BLER (Bridge Far)
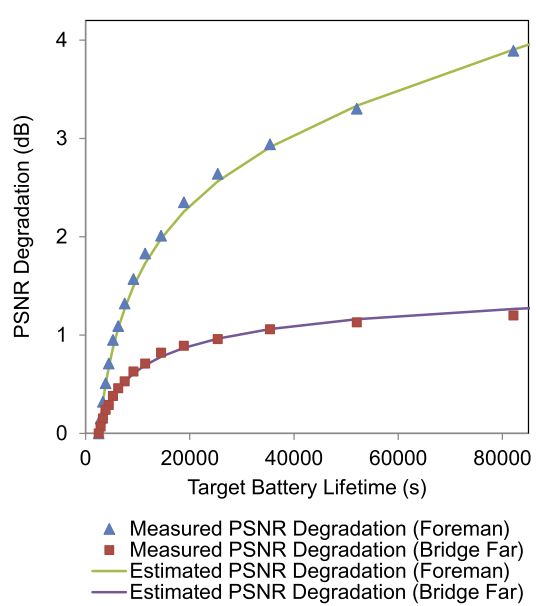

(b)

Fig. 7. Measurement results in battery lifetime-scalable mode with $\mathrm{P}_{\mathrm{EN}}=0$. (a) Lifetime error. (b) PSNR degradation. 

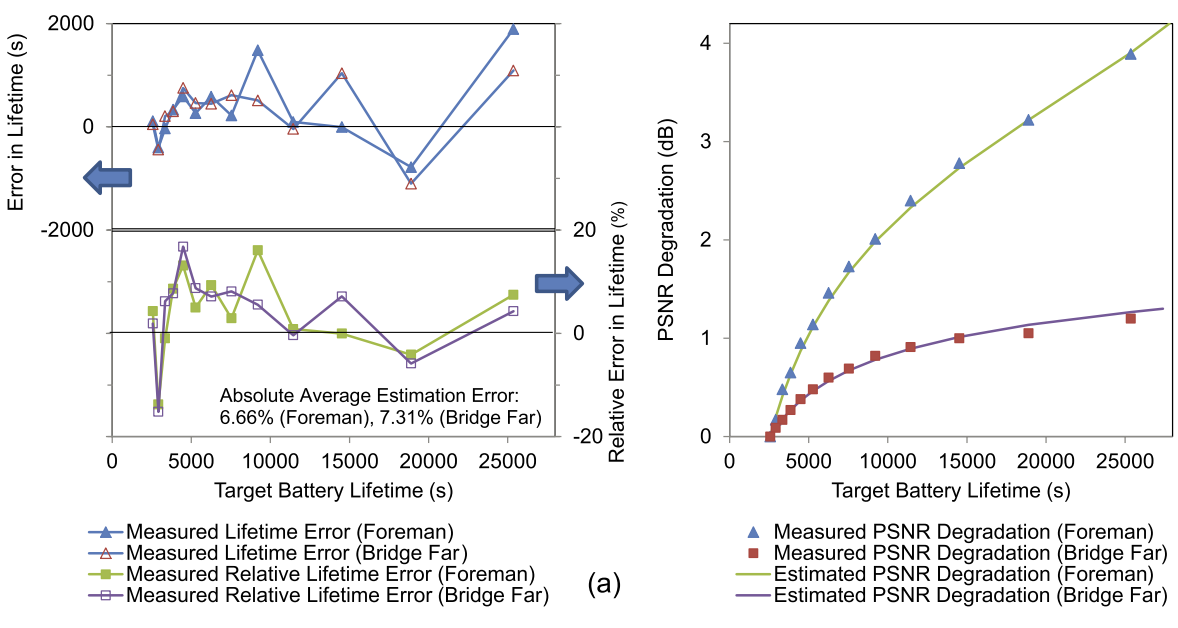

(b)

Fig. 8. Measurement results in battery lifetime-scalable mode with $\mathrm{P}_{\mathrm{EN}}=0.2$. (a) Lifetime error. (b) PSNR degradation.

Figs. 7(a) and (b) show the lifetime estimation error and PSNR degradation with $\mathrm{P}_{\mathrm{EN}}=0$. Absolute average estimation error of lifetime extension was less than $6.70 \%$. Measured PSNR quality was degraded by $1.20 \mathrm{~dB}$ (Bridge Far) $3.89 \mathrm{~dB}$ (Foreman). Figs. 8(a) and (b) show the lifetime estimation error and PSNR degradation with $\mathrm{P}_{\mathrm{EN}}=0.2$. Absolute average estimation error of lifetime extension was less than $7.31 \%$. Measured PSNR quality was degraded by $1.20 \mathrm{~dB}$ (Bridge Far) 3.89 dB (Foreman).

From the measurement results, battery lifetime is fully scalable and precisely controlled to keep target constraint. PSNR degradation is also precisely estimated. When encoder power increases, PSNR quality is more degraded at the same battery lifetime, since encoder power is not changed by LQSVT. Note that maximum PSNR degradation is same in $\mathrm{P}_{\mathrm{EN}}=0$ and $\mathrm{P}_{\mathrm{EN}}=0.2$, but this is due to the bitrate limitation of $\mathrm{BR}_{\mathrm{TAR}} \geq 62.5 \mathrm{kbps}$.

\section{Conclusion}

A battery lifetime and PSNR quality-scalable video transmission is proposed based on the battery recovery effect. Both battery lifetime and PSNR quality are fully scalable, and battery lifetime is significantly extended at the cost of PSNR degradation. Simple but precise estimation models are proposed for PSNR degradation and battery lifetime. Experimental results show that the proposed method significantly extends battery lifetime at the cost of acceptable PSNR degradation with precise and scalable battery lifetime and PSNR quality controls.

\section{Acknowledgments}

This research was supported by the Industrial Core Technology Development Program (10049095, "Development of Fusion Power Management Platforms and Solutions for Smart Connected Devices") funded by the Ministry of Trade, Industry \& Energy, Korea. 